\title{
Clinical manifestations of Whipple's disease mimicking rheumatic disorders
}

\author{
Eugeniusz J. Kucharz ID , Justyna Kramza ID , Anida Grosicka ID, Robert Pieczyrak ID \\ Department of Internal Medicine, Rheumatology and Clinical Immunology, Medical University of Silesia, Katowice, Poland
}

\begin{abstract}
Whipple's disease is a rare, chronic, systemic disorder caused by Tropheryma whipplei infection. The most common symptoms are weight loss, arthralgia, diarrhea and abdominal pain. Other organ involvement can also occur in the patients. Joint manifestations may mimic rheumatoid arthritis or spondyloarthritis. Arthalgia, arthritis, spondylodiscitis, bursitis and/or tenosynovitis are seen in the majority of the patients. This explains why some of the symptoms are misdiagnosed as those of rheumatic diseases. Understanding of Whipple's disease is important for differential diagnostics of several rheumatic symptoms.
\end{abstract}

Key words: Whipple's disease, arthritis, spondylodiscitis.

\section{Introduction}

Whipple's disease (WD) is a rare multisystem infectious disease caused by the bacterium Tropheryma whipplei. The disease involves principally the duodenum and the rest of the small intestine, heart, brain, and joints but can affect almost all organs. Humans are the only known host of Tropheryma whipplei. Whipple's disease is a chronic, progressive condition and may be fatal in untreated individuals.

Whipple's disease is characterized by a plethora of symptoms and signs as well as a heterogenous clinical pattern of the disease course. Almost all patients suffer from the articulo-muscular involvement which is akin to manifestations of common rheumatic disorders. Thus understanding of WD seems to be important for rheumatological practice. Published case reports confirmed occurrence of misdiagnosis of WD resulting in administration of an inappropriate antirheumatic medication.

The review is based on the PubMed database with the key term "Whipple disease" as the main search term. Additionally, the term "Tropheryma whipplei" was searched, and papers which refer to the clinical picture of the disease were used. Selected papers found searched with the term "differential diagnosis of arthritis" were also applied for the review.
The first description of a patient suffering from WD was included in the paper by Allchim and Hebb [1] which appeared in print in 1895 . The authors of the report did not recognize the described case as a new disease and referred to the condition as "lymphangiectasis intestini".

In 1907, the great American physician, pathologist and medical school educator George Hoyt Whipple (1878-1976) for the first time described WD as a separate nosological entity. He named a new disorder, intestinal lipodystrophy [2]. He did not recognize bacterial etiology of the disease and reported the disease as a condition caused by disturbed lipid metabolism. On the other hand, he did report a "silver-stained rod-shaped organism, closely resembling the tuberculosis bacillus" in the vacuoles of macrophages of patients with WD.

Black-Schaffer [3] in 1949 summarized histological findings in WD and used periodic acid-Schiff reagent to stain inclusions in macrophages obtained from the intestinal tissue and mesenteric lymph nodes. It was the first histopathological criterion of WD.

The first suggestion of infectious etiology of the disease appeared in 1952 when successful management with chloramphenicol was reported [4]. In 1960, free rodshaped bodies were detected with electron microscopy in the lamina propria of the intestine of patients with WD $[5,6]$. Bacterial etiology was considered but despite

\footnotetext{
Address for correspondence:

Eugeniusz J. Kucharz, Department of Internal Medicine, Rheumatology and Clinical Immunology, Medical University of Silesia, 45/47 Ziołowa St., 40-635 Katowice, Poland, e-mail: ejkucharz@poczta.onet.pl, ORCID: http://orcid.org/0000-0003-0924-2260 Submitted: 20.01.2021; Accepted: 31.03.2021
} 
the efficacy of antibiotics, the observed particles were also suggested to be virus-like particles. Further evaluation was done with PCR amplification of bacterial RNA, and later the causative bacterium was named Tropheryma whippelii $[7,8]$.

The name was coined from Greek words

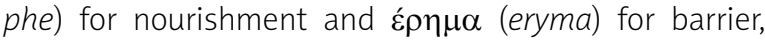
i.e. causing malabsorption. The bacterium was renamed Tropheryma whipplei in 2001 [9] because the earlier name had a spelling error. Attempts to culture the bacterium or to transfer infection to experimental animals were for years unsuccessful. In 1997, Schoedon et al. [10] cultured Tropheryma whipplei in vitro using deactivated mononuclear phagocytes. Later, the bacterium was successfully cultured in vitro using human fibroblast cell lines [11].

It should be mentioned that George Hoyt Whipple, a Nobel Prize laureate (1934), was unrelated to Allen Oldfather Whipple (1881-1963), a physician and pioneer in modern surgery who described the Whipple procedure and Whipple triad. Both of them were, however, lifelong friends [12].

An interesting fact is included in the paper of Sharma [13]. He made a profound analysis of records which characterized symptoms of disease of Ludwig van Beethoven, and concluded that most of the symptomatology matched WD. His interpretation of medical aspects of the life of Beethoven enriched a long list of suggested diagnoses of the great musician's illness.

\section{Epidemiology}

The epidemiology of WD remains unclear. It is a rare disease. Most of the papers used data provided by Dobbins [14] in 1987. The annual incidence of the disease based on investigation of duodenal biopsy specimens was about 12 new cases worldwide. This estimation was limited to the classic subset of WD. Introduction of PCR testing in 1991 revealed the relatively large number of cases. Cases reported in print (363 patients) in 1991-2001 were summarized by Dutly and Altwegg [15].

Most of the reported cases revealed that WD affects predominantly Caucasian males. Early observations indicated a male-to-female ratio of about $8: 1$. Recent data suggested a smaller difference between sexes in disease occurrence. Predominance of Caucasian patients remains unclear, and was suggested to result from different access to health care in various regions of the world. This suggestion is supported by studies on carriage in Asia and Africa $[16,17]$.

In 2015, Italian research estimated prevalence of the disease in north-western Italy at 3/1 000000 [18]. Epidemiological study from the USA covering the national data between 2012 and 2017 revealed prevalence of $9.8 / 1000000$ [19]. It is almost ten times more than the often quoted prevalence of 1/1 000000 [20]. Poor personal hygiene and contact with wastewaters are suggested to be risk factors of infection.

The disease may affect individuals of all ages. A review of cases from 1991-2001 revealed mean age of 51 (range, 4 to 77 years) at the onset of the clinically overt disease [21]. The older age of the patients, especially those reported earlier, resulted from a significant delay between initial symptoms and final diagnosis.

Introduction of more sensitive biomolecular methods of the disease detection facilitated diagnosis of a relatively high number of asymptomatic cases [20]. Carriage of Tropheryma whipplei seems to be an important problem in epidemiology of WD. Relatives of patients with chronic WD are commonly carriers, and facilitate transmission of the disease. It is believed that human-tohuman transmission is the most important route of the disease spread. Symptomatic patients have a high bacterial load in stool but asymptomatic carriers also have positive stool samples [21].

The bacterium was found in other body fluids including saliva and urine. It is believed that the bacterium is relatively common. Serum antibodies against Tropheryma whipplei are found in up 2/3 of the general population in selected regions of the world. Asymptomatic carriers of the bacterium can represent a large reservoir for other individuals. It supports the concept that the disease becomes overt only in genetically susceptible individuals in whom colonization of the gastrointestinal system occurs [20].

The role of nonhuman sources of Tropheryma whipplei remains unclear. Some observations suggest a passive role of domestic or synanthropic animals in distribution of bacteria. There is a single report of a gorilla affected by symptoms akin to WD [22]. The disease known as histiocytic ulcerative colitis in dogs is similar to WD but bacterial etiology of the disease has never been evidenced [23].

\section{Pathogenesis}

Tropheryma whipplei is a rod-shaped bacterium. It is approximately $2 \mathrm{~mm}$ long and $0.25-0.5 \mathrm{~mm}$ in diameter [24]. The bacterium has an unusual trilaminar cell wall detectable with electron microscopy. The wall consists of an inner layer of polysaccharides, a middle electron-translucent layer and an outer layer. It has been suggested that the outer layer is of host origin when the bacterium occurs within the cell [24]. Genetic studies supported the view that the bacteria is not closely related to other bacterial species. Further molecular investigation revealed that Tropheryma whipplei is phylogenetically located between the actinomycetes with group B peptidoglycan and Cellulomonadaceae. Similari- 
ties in bacterial RNA with such species as Mycobacterium konsasii, Mycobacterium gastri, Mycobacterium malmoense and Aeromonas trota have been detected [25].

Tropheryma whipplei does not grow on in vitro cultures. Intracellular growth of the bacterium was achieved in a human fibroblast cell line. It has been calculated that the doubling time for the bacteria in vitro is relatively long, that is about 18 days [24].

Genetic factors of the host are considered as a main factor for susceptibility and facilitate development of familial clusters of cases. It is suggested that the genetic pattern of regulatory genes responsible for the expression of inflammatory cytokines predisposes to the infection [21, 24].

The role of the immune system in development of clinically overt disease is very important. In the presence of a fully functional immunity, infection may be eliminated or is limited to asymptomatic colonization of the gastrointestinal tract. Most commonly, Tropheryma whipplei infected the small intestine. The bacterium creates an anti-inflammatory milieu. The bacterium is phagocytosed by macrophages but the cells are unable to kill the internalized bacteria due to ability of the bacteria to reduce the inflammatory response. This phenomenon is also associated with inappropriate antigen presentation and impaired maturation of macrophagic phagosomes. All these phenomena resulted in diminution of the $T$ cell response and an impaired immune response to infection [26]. Additionally, production of specific immunoglobulins is very low. Several of the mechanisms involved in impaired pathogen clearance in patients with WD are suggested to be responsible for development of joint inflammation. It may explain the common occurrence of arthritis in patients suffering from WD.

\section{Clinical manifestations}

Whipple's disease is characterized by a heterogeneous clinical picture and a plethora of symptoms and signs. Dolmans et al. [27] classified symptoms and signs of the disease in common and less common as well as within each group into those related to the gastrointestinal system and those which are extraintestinal manifestations. Other studies categorized the clinical subsets of the disease as classic WD, acute infection, and localized chronic infection [15].

According to the first classification of the disease manifestations, the common signs and symptoms related to the gastrointestinal system are weight loss (80-90\%), diarrhea (70-85\%) and abdominal pain (50-90\%). Common extraintestinal manifestations are arthralgia and arthritis (70-90\%), anemia (75-90\%), low grade intermittent fever (40-60\%), lymphadenopathy (40-60\%) and hyperpigmentation (40-60\%). Less common gastrointestinal manifestations are abdominal mass and hematochezia. Less common extraintestinal manifestations are related to the cardiovascular system (pericardial friction rub, murmurs, and conduction abnormalities detectable with ECG), pulmonary system (long-lasting cough, pleuritic pain), central nervous system (cognitive changes, supranuclear gaze palsy, altered level of consciousness, ataxia, and sensory deficits), and ocular (visual loss, uveitis, and retinitis). Splenomegaly and ascites were found in 5-10\% of the patients [27].

The classic course of WD consists of the early phase ( $<6$ years) (intermittent arthralgia and fever), the middle phase (6-8 years) featuring diarrhea with chronic obstructive troubles, weight loss, and abdominal pain, and the late phase ( $>8$ years) with predominant neurological symptoms [28].

Neurological manifestations are characterized by a variety of symptoms and signs, and can be severe. In the majority of the patients, neurological manifestations are associated with other involvements. Isolated neurological pattern of the disease is uncommon. Central nervous system symptoms include cognitive disturbances, pyramidal and extrapyramidal symptoms, headaches, sleep disorders, cerebellar palsy, abnormal involuntary movements, epilepsy, and oculomotor nerve palsy [28]. In rare cases, WD can present as cystic brain tumor [29]. Progressive dementia may also be a pattern of WD $[30,31]$. Most of these symptoms need to be differentiated from various forms of systemic vasculitides.

Acute infections are characterized by pneumonia and gastroenteritis and chronic localized infections are shown as endocarditis and encephalitis. Infections in other locations are less common [27].

\section{Musculoskeletal manifestations}

Symptoms and signs associated with the joints, muscles and bones are the most common extraintestinal manifestations of WD (Table I). Arthralgia is a very common symptom seen in the early phase of the infection. It is a part of the classic clinical pattern of the disease. Seronegative arthritis and/or arthralgia are prodromal symptoms in about 80-90 per cent of cases. This stage of the disease is frequently associated with elevated acute-phase reactants. The majority of patients have prolonged joint symptoms during the course of the disease. Cumulative analysis of symptomatology of the patient cohort revealed "rheumatological symptomatology" in 60 to 90 per cent of patients [27]. Early arthritis was found to be detectable for an average of 6.7 years before the final diagnosis of infection [32]. Arthritis is usually migrating, i.e. characterized by palindromic pattern [33, 34]. It may be useful in differential diagnosis with rheumatoid arthritis. 
On the other hand, most of the patients are misdiagnosed with more common forms of arthritides. Rosa da Silva et al. [35] reviewed cases of WD akin to other polyarthralgias. Glaser et al. [36] reported 7 patients who had been referred to the rheumatological center with diagnosis of rheumatoid arthritis and had been receiving medication for this disease. All of them later were found to suffer from WD. Six of seven of them were male. All suffered from polyarthritis with a predominantly symmetrical pattern. Five of seven had involvement of the wrists, metacarpophalangeal joints and knees. Serological indices (rheumatoid factor, ACPA antibodies) were absent. Inflammatory indices were elevated. All patients fulfilled the EULAR/ACR criteria for rheumatoid arthritis. Seronegative arthritis was of moderate activity, and median DAS28 at initial presentation was 4.3. The patients received treatment including disease modified antirheumatic drugs, glucocorticoids and biologic agents. All patients showed an insufficient response to the medication. It is of interest that extraarticular symptoms in the patients, especially gastrointestinal ones, were mild and were attributed to adverse reactions of the medication. Diagnosis was performed with PCR detection of the bacterial DNA in the synovial fluid $[35,36]$.

Chronic bursitis and tenosynovitis can be predominant manifestations of WD. Alterations are usually located at several sites of the body. Medication with methotrexate and etanercept was found to be ineffective [37].

The palindromic rheumatism pattern of WD can mimic infectious arthritis and is characterized by sudden development of swelling, pain and redness accompanied by loss of function [34].

Whipple's disease in a few patients mimics spondyloarthropathy. Back pain is the most common symptom. Imaging techniques may reveal inflammation and destruction of the sacroiliac joints [38]. Coexistence of ankylosing spondylitis and WD was also reported [39]. Deformations of the spine are rare. Non-steroidal antirheumatic drugs are ineffective and administration of TNF- $\alpha$ inhibitors usually aggravated the disease and facilitated making the WD diagnosis [40-44]. It is of note that Tropheryma whipplei can cause uveitis, a common extraarticular manifestation of inflammatory spondyloarthropathy [45] (Table I).

Other manifestations akin to rheumatic disease have also been reported, including oculomotor myopathy [46], osteomyelitis [47], and cervical spine abnormalities [48]. Spondylodiscitis is not so rare in patients with WD, and may be an initial symptom $[49,50]$. A case of WD associated with psoriatic arthritis was reported as well [51].

\section{Diagnosis}

Whipple's disease is a rare condition and is diagnosed frequently at a late stage. The disease is charac-
Table I. Symptoms of Whipple's disease resembling those of rheumatic diseases (from various sources)

\begin{tabular}{|c|}
\hline Very common \\
\hline Polyarthralgia (migrating, rapid onset, episodic attacks) \\
\hline $\begin{array}{l}\text { Polyarthritis (symmetric, migrating, rarely leading to } \\
\text { deformations) }\end{array}$ \\
\hline $\begin{array}{l}\text { The most commonly involved joints: wrist, knee, ankle, hip, } \\
\text { shoulder, proximal and distal interphalangeal joints }\end{array}$ \\
\hline Back pain (inflammatory) \\
\hline Fever (low grade) \\
\hline Fatigue \\
\hline Common \\
\hline Myalgia (various localization) \\
\hline Tenosynovitis \\
\hline Bursitis \\
\hline $\begin{array}{l}\text { Cutaneous manifestations (subcutaneous nodules, purpura, } \\
\text { hyperpigmentation) }\end{array}$ \\
\hline Rare \\
\hline Pleural effusions \\
\hline Pulmonary infiltrations \\
\hline Pericardial friction rub \\
\hline Spondylodiscitis \\
\hline Isolated sacroiliac joint involvement \\
\hline
\end{tabular}

terized by a variety of nonspecific symptoms and signs. Additionally, common administration of antibiotics due to various reasons may cause alterations in clinical presentation of the disease. It is also important to consider WD as a possible factor that mimics symptoms of rheumatic disorders.

El-Abassi et al. [24] presented in 2017 a diagnostic strategy for the classic subset of WD. Application of the strategy is suggested primarily for patients with chronic diarrhea or abdominal pain but in our opinion can be applied to cases of unclear arthritis with nonspecific systemic symptoms as well. Especially patients resistant to standardized medication administered in rheumatoid arthritis or inflammatory spondyloarthropathy should be considered. The first step of diagnostics includes PCR screening of saliva and feces samples. In both positive and negative results the second diagnostic step is recommended, duodenal biopsy with PAS staining of multiple specimens.

Positive PCR and duodenal biopsy histopathological findings result in diagnosis of definite WD. Obtaining only one positive test (either PCR or duodenal biopsy) indicates the need for further diagnostics, including immunohistochemically analysis of the tissue sample. Negative results exclude the classic subset of WD but localized WD still can be considered [24]. 
It should be mentioned that bacteria are not distributed equally in all parts of the intestine. Additional biopsy of the gastric antrum, jejunum or ileum is needed in selected patients. Sampling of joint mucosal membrane is a less commonly applied diagnostic method. Application of electron microscopy may enhance sensitivity of the biopsy evaluation. Serology is also an alternative nonstandardized diagnostic tool [24].

A broad spectrum of symptoms and signs of WD is associated with differential diagnosis. It should include a number of disorders of various etiology [52-54]. A key for diagnosis is evidence of infection with the causative microorganism.

\section{Treatment}

The main target of medication is eradication of Tropheryma whipplei. Antibiotics are drugs of choice but there are no uniform guidelines for the kind of management, and various therapeutic regimens are suggested. El-Abassi et al. [24] summarized current treatment recommendations. In patients with the classic subset of WD one year of combined medication with doxycycline (200 mg/24 h, orally) and hydroxychloroquine (600 mg/24 h, orally) is recommended. This medication is to be continued with $200 \mathrm{mg}$ daily of doxycycline only for lifetime.

Patients with the central nervous system involvement are recommended to be given ceftriaxone ( $2 \mathrm{~g}$ intravenously) every $12 \mathrm{~h}$ for 6 weeks, followed by cotrimazole (800 mg/160 mg every $12 \mathrm{~h}$, orally) for 1 year.

Localized WD, including articular involvement, is to be treated with doxycycline and hydroxychloroquine in the same dose as the classical subset of WD but for 12-18 months with lifetime follow-up with doxycycline. There are suggestions to use PCR for follow-up monitoring of the treatment [24]. The antibiotics used for a lifetime follow-up are relatively safe but the safety and effectiveness of such medication still have no clear evidence. Effectiveness of the therapy depends on stage of the disease. Relapsing disease was reported [55].

Most of the studies on therapy of WD indicated the negative role of immunosuppression. Reported cases suggested association of initiation of immunosuppression with onset of diarrhea and subsequent diagnosis of WD. TNF- $\alpha$ inhibitors have been shown to result in fatal exacerbation of the disease [40-44]. Previous immunosuppressive therapy can be associated with the development of immune reconstitution inflammatory syndrome in patients with WD. It has been reported that Tropheryma whipplei exerts suppression on CD4+ T-cells and the syndrome can develop after antibiotic medication [20]. Glucocorticoids are drugs of choice in patients with immune reconstitution inflammatory syndrome [56].

\section{Conclusions}

Whipple's disease results from infection with Tropheryma whipplei in individuals with a predisposition (probably genetic) to development of the bacterial colonization. The disease has a plethora of symptoms, including articular, osseous and muscular involvement. It results in a variety of clinical presentations, some of which can mimic various rheumatic disorders. Rheumatic patients with an atypical course of disease and resistant to medication should be considered in differential diagnosis for WD. Understanding of the clinical picture and diagnosis of WD is important for rheumatologists.

The authors declare no conflict of interest.

\section{References}

1. Allchin WH, Hebb RG. Lymphangiectasis intestine. Trans Path Soc (London) 1895; 46: 221-223.

2. Whipple GH. A hitherto undescribed disease characterized anatomically by deposits of fat and fatty acids in the intestinal and mesenteric lymphatic tissues. Bull Johns Hopkins Hosp 1907; 18: 3582-391.

3. Black-Schaffer B. The tinctorial demonstration of a glycoprotein in Whipple's disease. Proc Soc Exp Biol Med 1949; 72: 225-227.

4. Paulley JW. A case of Whipple's disease (interstinal lipodystrophy). Gastroenterology 1952; 22: 128-132.

5. Cohen AS, Schimmel EM, Holt PR, Isselbacher KJ. Ultrastructural abnormalities in Whipple's disease. Proc Soc Exp Biol Med 1960; 105: 411-414, DOI: 10.3181/00379727-105-26126.

6. Chears WC, Ashwortj CT. Electron microscopy study of the interstitial mucosa in Whipple's disease: demonstration of encapsulated bacilliform bodies in the lesion. Gastroenterology 1961; 41: 129-138.

7. Wilson KH, Blitchington R, Frothingham R, Wilson JA. Phylogeny of the Whipple's-disease-associated bacterium. Lancet 1991; 338: 474-475, DOI: 10.1016/0140-6736(91)90545-z.

8. Relman DA, Schmidt TM, MacDermott RP, Falkow S. Identification of the uncultured bacillus of Whipple's disese. N Engl JMed1992;327:293-301,DOI:10.1056/NEJM199207303270501.

9. La Scola B, Fenollar F, Fournier PE, et al. Description of Tropheryma whipplei gen. nov., sp. nov., the Whipple's disease bacillus. Int J Syst Evol Microbiol 2001; 51 (Pt 4): 1471-1479, DOI: 10.1099/00207713-51-4-1471.

10. Schoedon G, Goldenberger D, Forrer R, et al. Deactivation of macrophages with IL-4 is the key to isolation of Tropheryma whippelii. J Infect Dis 1997; 176: 672-677, DOI: 10.1086/514089.

11. Raoult D, Birg ML, La Scola B, et al. Cultivation of the bacillus of Whipple's disease. N Engl J Med 2000; 342: 620-625, DOI: 10.1056/NEJM200003023420903.

12. Wysocki WM, Komorowski AL, Wysocki A. Jedno nazwisko, dwóch lekarzy, czyli o metodzie Whipple'a i chorobie Whipple'a. Przegl Lek 2005; 62: 139-140.

13. Sharma OP. Beethoven's illness: Whipple's disease rather than sarcoidosis? J Roy Soc Med 1994; 87: 283-285. 
14. Dobbins WO III. Whipple's disease. Charles C Thomas, Springfield IL 1987.

15. Dutly F, Altwegg M. Whipple's disease and Tropheryma whippelii. Clin Microbiol Rev 2001; 14: 561-583, DOI: 10.1128/ CMR.14.3.561-583.2001.

16. Keita AK, Mediannikov O, Ratmonov P, et al. Looking for Tropheryma whipplei source and reservoir in rural Senegal. Am J Trop Med Hyg 2013; 88: 339-343, DOI: 10.4269/ajtmh.2012.12-0614

17. Keita AK, Dubot-Pérès A, Phommasone K, et al. High prevalence of Tropheryma whipplei in Lao kindergarten children. PLoS Negl Trop Dis 2015; 9: e0003538, DOI: 10.1371/journal. pntd.0003538.

18. Biagi F, Balduzzi D, Delvino P, et al. Prevalence of Whipple's disease in north-western Italy. Eur J Clin Microbiol Infect Dis 2015 . 34: 1347-1348, DOI: 10.1007/s10096-015-2357-2.

19. Elchert JA, Mansoor E, Abou-Saleh M, Cooper GS. Epidemiology of Whipple's disease in the USA between 2012 and 2017 a populations-based national study. Dig Dis Sci 2019; 64 1305-1311, DOI: 10.1007/s10620-018-5393-9.

20. Schneider T, Moos V, Loddenkemper C, et al. Whipple's disease: new aspects of pathogenesis and treatment. Lancet Infect Dis 2008; 8: 179-190, DOI: 10.1016/S1473-3099(08)70042-2.

21. Misbah SA, Mapstone NP. Whipple's disease revisited. J Clin Pathol 2000; 53: 750-755, DOI: 10.1136/jcp.53.10.750.

22. Van Kruiningen HJ, Dobbins WO, John G. Bacterial histiocytic colitis in a lowland gorilla (Gorilla Gorilla gorilla). Vet Patho 1991; 28: 544-546, DOI: 10.1177/030098589102800616.

23. Van Kruiningen HJ, Montali RJ, Strandberg JD, Kirk RW. A granulomatous colitis of dogs with histological resemblance of Whipple's disease. Pathol Vet 1965; 2: 521-544, DOI: 10.1177/ 030098586500200601.

24. El-Abassi R, Soliman MY, Williams F, England JD. Whipple's disease. J Neurol Sci 2017; 377: 197-206, DOI: 10.1016/j.jns.2017. 01.048.

25. Maiwald M, Ditton A, von Herbay FA, et al. Reassessment of the phylogenetic position of the bacterium associated with Whipple's disease and determination of the 16S-23S ribosomal intergenic spacer sequence. Int J Syst Bacteriol 1996; 46: 1078-1082, DOI: 10.1099/00207713-46-4-1078.

26. Berthelot J-M, Puéchal X. Impaired intracellular pathogen clearance and inflammatory joint disease: is Whipple's dis ease a guiding light? Joint Bone Spine 2018; 85: 531-536, DOI 10.1016/j.jbspin.2017.09.007

27. Dolmans RAV, Boel CH, Lacie MM, Kusters JG. Clinical manifestation, treatment, and diagnosis of Tropheryma whipple infections. Clin Microbiol Rev 2017; 30: 529-553, DOI: 10.1128/ CMR.00033-16

28. Compain C, Sacre K, Puéchal X, et al. Central nervous system involvement in Whipple disease. Clinical study of 18 patients and long-term follow-up. Medicine (Baltimore) 2013; 92: 324-330, DOI: 10.1097/MD.0000000000000010.

29. Kilani M, Njim L, Nsir AB, Hattab MN. Whipple disease presenting as cystic brain tumor case report and review of the literature. Turk Neurosurg 2018; 28: 495-499, DOI: 10.5137/10195149.JTN.17111-16.2.

30. Hurth K, Tarawneh R, Ghoshal N, et al. Whipple's disease mas querades as dementia with Lewy bodies. Alzheimer Dis Assoc Disord 2015; 29: 85-89, DOI: 10.1097/WAD.0b013e3182a715da.
31. Chandra SR, Raj P, Pai AR, Reddy N. A case of Whipple's disease: a very rare cause for rapidly progressive dementia. Indian J Psychol Med 2018; 40: 280-283, DOI: 10.4103/IJPSYM. IJPSYM 14917.

32. Puéchal $X$. Whipple disease and arthritis. Curr Opin Rheumatol 2001; 13: 74-79, DOI: 10.1097/00002281-200101000-00012.

33. Krol CG, de Meijer PH. Palindromic rheumatism consider Whipple's disease. Int J Rheum Dis 2013; 16: 475-476, DOI: 10.1111/1756-185X.12084

34. Puéchal X. Whipple's disease. Ann Rheum Dis 2013; 72: 797803, DOI: 10.1136/annrheumdis-2012-202684.

35. Rosa da Silva GA, Pires Neto JS. Whipple's disease manifested as difficult-to-diagnose polyarthralgia: a case report and literature review. Rev Bras Rheumatol (England Ed) 2017; 57 : 483-48, DOI: 10.1016/j.rbre.2015.05.003.

36. Glaser C, Rieg S, Wiech T, et al. Whipple's disease mimicking rheumatoid arthritis can cause misdiagnosis and treatment failure. Orphanet J Rare Dis 2017; 12: 99, DOI: 10.1186/s13023017-0630-4.

37. Dubost JJ, Couderc M, Mathien S, et al. Chronic bursitis and tenosynovitis revealing Whipple's disease. Joint Bone Spine 2020; 87: 481-482, DOI: 10.1016/j.jbspin.2020.01.010.

38. Feurle GE. Association of Whipple's disease with HLA-B27. Lancet 1985; 325: 1336, DOI: 10.1016/s0140-6736(85)92831-4.

39. Martins MDL, Abreu P, Caldeira A, et al. Whipple disease and ankylosing spondylitis - a case report. J Clin Rheumatol 2020, DOI: 10.1097/RHU.0000000000001466 [Online ahead of print].

40. Ramos JM, Pasquau F, Galipienso N, et al. Whipple's disease diagnosed during anti-tumor necrosis factor alpha treatment: two case reports and review of the literature. J Med Case Rep 2015; 9: 165, DOI: 10.1186/s13256-015-0632-6.

41. Estévez-Gil M, de Castro-Parga ML, Carballo-Fernandez C, et al. Whipple disease in a patient under anti-TNF-alpha therapy. Gastroenterol Hepatol 2016; 39: 334-335, DOI: 10.1016/j.gastrohep.2015.04.005.

42. Sparsa L, Fenollar F, Gossec L, et al. Whipple disease revealed by anti-TNF $\alpha$ therapy. Rev Med Interne 2013; 34: 105-109, DOI: 10.1016/j.revmed.2012.10.371 [Article in French]

43. Gaddy JR, Khan ZZ, Chaser B, Scofield RH. Whipple's disease diagnosis following the use of TNF-alpha blockade. Rheumatology (Oxford) 2012; 51: 946, DOI: 10.1093/rheumatology/ ker387.

44. Hoppé E, Masson C, Audran M, et al. Whipple's disease diagnosed during biological treatment for joint disease. Joint Bone Spine 2010; 77: 335-339, DOI: 10.1016/j.jbspin.2010.03.015.

45. Sève P, Kodjikian L, Adélaïde L, Jamilloux Y. Uveitis in adults: What do rheumatologists need to know? Joint Bone Spine 2015; 82: 308-314, DOI: 10.1016/j.jbspin.2015.06.002.

46. Parkash V, Mudhar HS, Wagnere BE, et al. Bilateral ocular myositis associated with Whipple's disease. Ocul Oncol Pathol 2017; 3: 17-21, DOI: 10.1159/000448622.

47. Walter R, Bachmann SP, Schaffner A, et al. Bone marrow involvement in Whipple's disease: rarely reported, but really rare? Br J Haematol 2001; 112: 677-679, DOI: 10.1046/j.13652141.2001.02648.x.

48. Pérez Álvarez Ál, Moris de la Tassa G. Cervical myelopathy as a form of presentation of Whipple disease. Neurología 2019; 35: 583-585, DOI: 10.1016/j.nrl.2019.03.007. 
49. Weber U, Morf MH, Gubler JG, et al. Spondylodiscitis as the first manifestation of Whipple's disease in a removal worker with chronic low back pain. Clin Rheumatol 2003; 22: 443446, DOI: 10.1007/s10067-003-0786-2.

50. Mirsbrunner-Erni R, Altwegg M, Diener PA, Vilinger PM. Whipple's disease with normal intestinal histology: rarity or reality? Schweiz Med Wschr 2000; 130: 1820-1826.

51. Whistance RN, Elfarouki GW, Vohra HA, Livesey SA. A case of Tropheryma whipplei infective endocarditis of the aortic and mitral valves in association with psoriatic arthritis and lumbar discitis. J Heart Valve Dis 2011; 20: 353-356.

52. Totschnig D, Seitz T, Zoufaly A, et al. Whipple's disease diagnosed in a patient with suspected sarcoidosis. Int J Infect Dis 2021; 22: S1201-9712(21)00271-X, DOI: 10.1016/j.ijid.2021. 03.053 [Online ahead of print].
53. Melas N, Amin R, Gyllemark P, et al. Whipple's disease: the great masquerader $-\mathrm{a}$ high level of suspicion is the key to diagnosis. BMC Gastroenterol 2021; 21: 128, DOI: 10.1186/ s12876-021-01664-1.

54. Feurle GE, Moos V, Stroux A, et al. Differential diagnostic value of rheumatic symptoms in patients with Whipple's disease. Sci Rep 2021; 11: 5980, DOI: 10.1038/s41598-021-85217-2.

55. Ruggiero E, Zurio A, Giantin V, et al. Relapsing Whipple's disease: a case report and literature review. Eur J Gastroenterol Hepatol 2016; 28: 267-270, DOI: 10.1097/MEG.0000000000000539.

56. Nabeya D, Kinjo T, Yamaniha K, et al. Use of steroids to treat anti-tumor necrosis factor $\alpha$ induced tuberculosis-associated immune reconstitution inflammatory syndrome: case report and literature review. Medicine (Baltimore) 2020; 99: e22076, DOI: 10.1097/MD.0000000000022076. 